\title{
Editorial
}

\section{Theory and Algorithms of Variational Inequality and Equilibrium Problems, and Their Applications}

\author{
Xie-ping Ding, ${ }^{1}$ Nan-jing Huang, ${ }^{2}$ Fu-quan Xia, \\ Qing-bang Zhang, ${ }^{3}$ and Qamrul Hasan Ansari ${ }^{4}$ \\ ${ }^{1}$ Sichuan Normal University, Chengdu, Sichuan 610066, China \\ ${ }^{2}$ Sichuan University, Chengdu, Sichuan 610064, China \\ ${ }^{3}$ Southwestern University of Finance and Economics, Chengdu, Sichuan 611130, China \\ ${ }^{4}$ Aligarh Muslim University, Aligarh 202 002, India
}

Correspondence should be addressed to Xie-ping Ding; dingxip@sicnu.edu.cn

Received 17 September 2014; Accepted 17 September 2014; Published 22 December 2014

Copyright (C) 2014 Xie-ping Ding et al. This is an open access article distributed under the Creative Commons Attribution License, which permits unrestricted use, distribution, and reproduction in any medium, provided the original work is properly cited.

The variational inequality problem is a general problem formulation that encompasses many mathematical problems, among others, including nonlinear equations, optimization problems, complementarity problems, and fixed point problems. Variational inequality is developed as a tool for the study of certain classes of partial deferential equations, economic equilibrium problems, and the pricing model of the option.

Equilibrium problems provide a mathematical framework which includes optimization, variational inequalities, fixed point and saddle point problems, and noncooperative games as particular cases. It has received an increasing interest mainly because many theoretical and algorithmic results developed for one of these models can be often extended to the others by using the unifying language.

Because of the importance and active impact of variational inequality and equilibrium problem in the nonlinear analysis and optimization, this special issue, focusing on most recent contributions, includes works on hemivariational inequality and multivalued variational inequality, the well-posedness and stability of equilibrium problems, the basic methods for showing the existence of solution of the equilibrium problem, the iterative approximations, and some applications to the abstract economy and portfolio selection, which are based on a strict international peer review procedure and our original proposal. A brief review of the papers is given as the following five topics.
(I) Variational Inequality and Complementarity Problem

(i) In the paper titled "On a class of variationalhemivariational inequalities involving upper semicontinuous set-valued mappings," G. Tang et al. apply the various coercivity conditions to show the existence of solutions and boundedness of the solution set for the variational-hemivariational inequalities involving upper semicontinuous set-valued mappings.

(ii) In the paper titled "A new system of multivalued mixed variational inequality problem," $\mathrm{Xi} \mathrm{Li}$ and Xue-song Li construct a perturbational $f$-projection algorithm for solving a system of multivalued mixed variational inequality problem and prove the existence of solutions and the convergence of iterative sequences.

(iii) In the paper titled "An improvement of global error bound for the generalized nonlinear complementarity problem over a polyhedral cone," $\mathrm{H}$. Sun et al. study an easier computed global error bound for the generalized nonlinear complementarity problem over a polyhedral cone.

(II) Well-Posedness and Stability of Equilibrium Problems

(i) In the paper titled "Levitin-Polyak well-posedness of an equilibrium-like problem in Banach spaces," R. Deng discusses the Levitin-Polyak well-posedness of 
an equilibrium-like problem in Banach spaces under suitable conditions.

(ii) In the paper titled "Existence and well-posedness for symmetric vector quasi-equilibrium problems," $\mathrm{K}$. Wang et al. study the existence of the solutions and sufficient conditions for the generalized LevitinPolyak well-posedness of symmetric vector quasiequilibrium problems.

(iii) In the paper titled "The property of the set of equilibria of the equilibrium problem with lower and upper bounds on Hadamard manifolds," Q.-B. Zhang and G. Tang study the existence of solutions and the essential stability of the solution set of the equilibrium problem with lower and upper bounds on Hadamard manifolds.

(III) KKM Theorem, Coincidence Theorem, and Applications

(i) In the paper titled "Class $\mathfrak{U}-K K M(X, Y, Z)$, general KKM type theorems, and their applications in topological vector space," G. Tang and Q. Zhang establish some generalized KKM theorems and apply them to show some fixed point theorems and existence theorems of solutions for the generalized vector equilibrium problems.

(ii) In the paper titled "Some new coincidence theorems in product GFC-spaces with applications," J. Zhao obtains a continuous selection theorem and applies to proof some new collective fixed point theorems and coincidence theorems in product GFC-spaces.

\section{(IV) Convergence Iterative Process for Mappings}

(i) In the paper titled "A strong convergence algorithm for the two-operator split common fixed point problem in Hilbert spaces," C.-C. Hong and Y.-Y. Huang investigate an iterative scheme to approximate the minimum norm solution of the two-operator split common fixed point problem with firmly nonexpansive mappings and compare the performance of the presented algorithm.

(ii) In the paper titled "Weak and strong convergence theorems for finite families of asymptotically quasinonexpansive mappings in Banach spaces," L. Deng and J. Xiao construct a finite-step iteration sequence for two finite families of asymptotically nonexpansive mappings and prove its weak and strong convergence in Banach space.

(iii) In the paper titled "Iterative schemes for convex minimization problems with constraints," L.-C. Ceng et al. analyze the strong convergence of an implicit iterative algorithm for finding a solution of the minimization problem for a convex and continuously Fréchet differentiable functional with constraints of the generalized mixed equilibrium problem, the system of generalized equilibrium problems, and finitely many variational inclusions in real Hilbert spaces. (iv) In the paper titled " $A$ continuous trust-region-type method for solving nonlinear semidefinite complementarity problem," Y. Ji et al. propose a new method to solve nonlinear semidefinite complementarity problem by combining a continuous method and a trustregion-type method and show the well-definedness of the method and its global convergence.

(V) Applications to the Abstract Economy and Portfolio Selection

(i) In the paper titled "Bounded rationality of generalized abstract fuzzy economies," by using a nonlinear scalarization technique, L. Wang and Y. Fu study the bounded rationality model for generalized abstract fuzzy economies and prove the structural stability and robustness to $(\lambda, \varepsilon)$-equilibria of generalized abstract fuzzy economies in finite continuous spaces.

(ii) In the paper titled "Continuous-time mean-variance portfolio selection under the CEV process," $\mathrm{H}$. Ma derives an optimal portfolio strategy and the efficient frontier of continuous-time mean-variance portfolio selection model and gives some numerical examples.

\section{Acknowledgments}

The editors of this special issue would like to express their gratitude to the authors who have submitted their papers for consideration and to the expert referees who provided them with invaluable help in the reviewing process.

Xie-ping Ding Nan-jing Huang

Fu-quan Xia

Qing-bang Zhang

Qamrul Hasan Ansari 


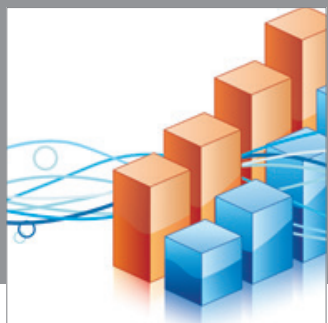

Advances in

Operations Research

mansans

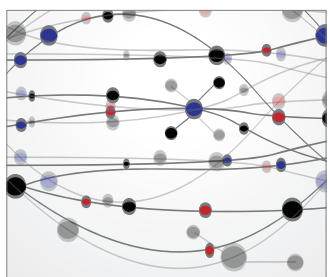

The Scientific World Journal
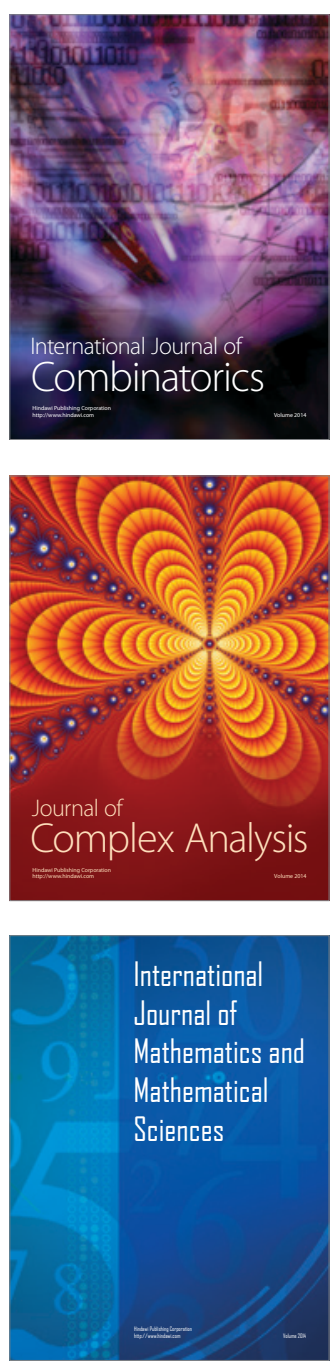
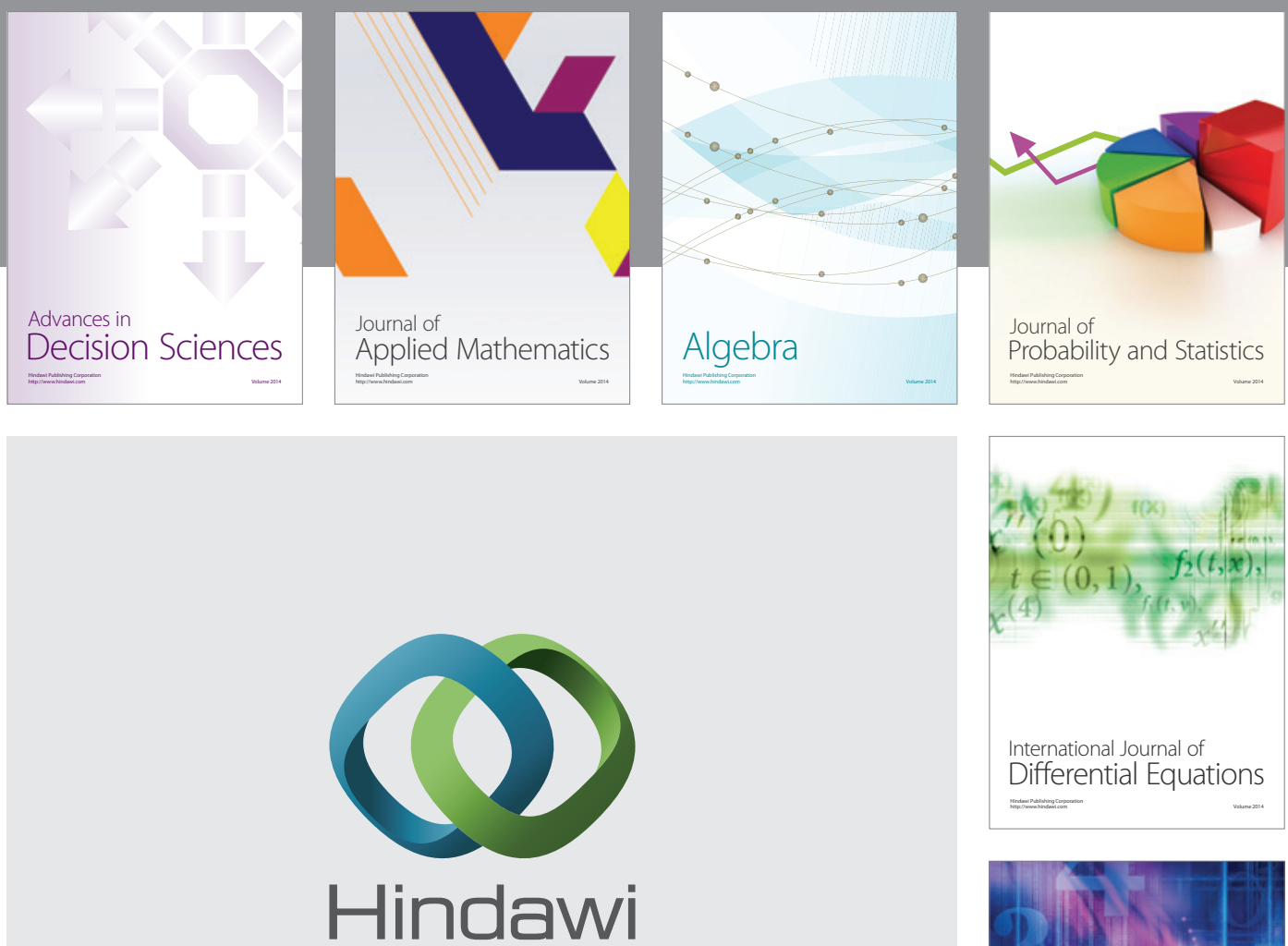

Submit your manuscripts at http://www.hindawi.com
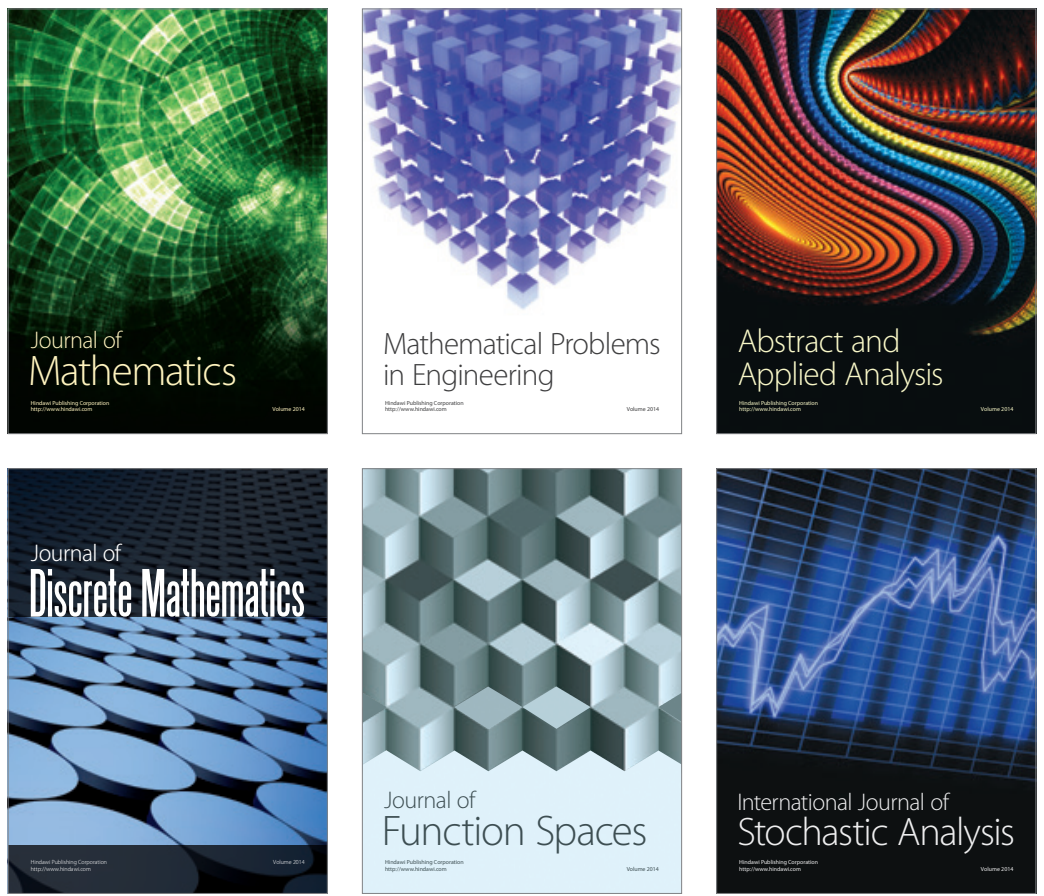

Journal of

Function Spaces

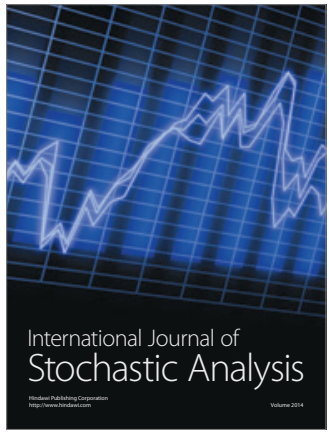

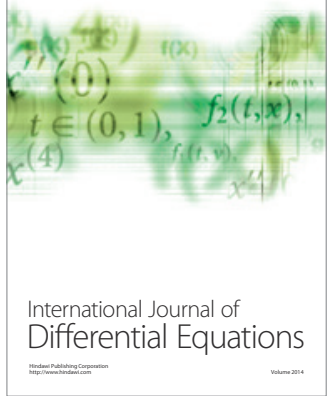
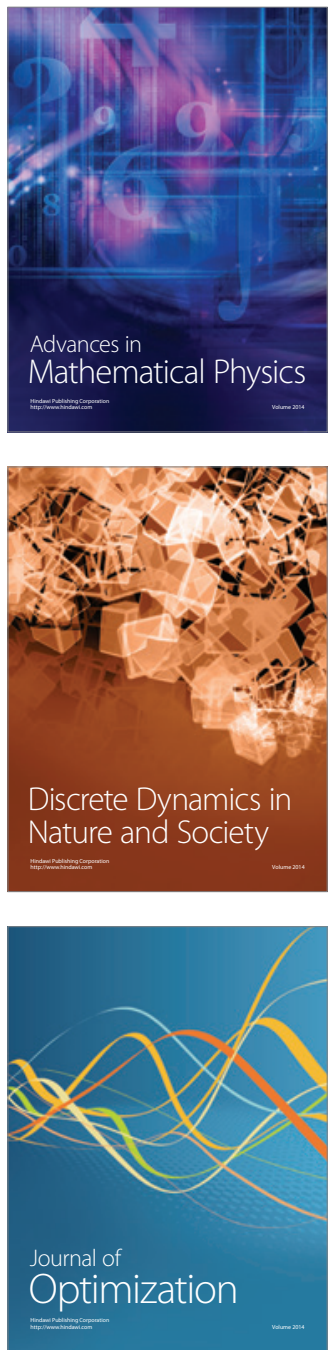